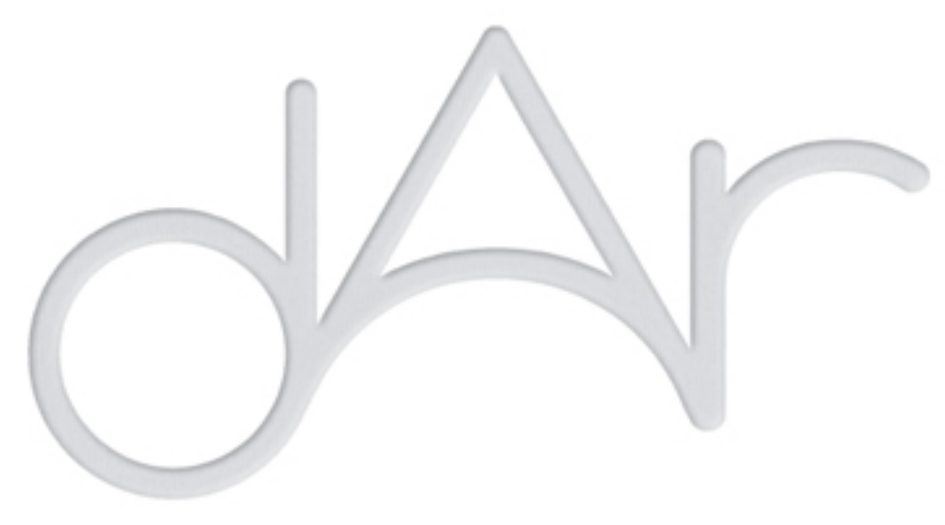

\title{
Développement d'un écosystème territorial d'innovation sociale dans les zones touristiques vulnérables au Maroc: cas des territoires oasiens
}
Autor(es):
Askour, Khadija
Publicado por: Imprensa da Universidade de Coimbra
URL persistente:
URI:http://hdl.handle.net/10316.2/44422
DOI:
DOI:https://doi.org/10.14195/2182-844X_5_1
Accessed : $\quad$ 26-Apr-2023 03:15:27

A navegação consulta e descarregamento dos títulos inseridos nas Bibliotecas Digitais UC Digitalis, UC Pombalina e UC Impactum, pressupõem a aceitação plena e sem reservas dos Termos e Condições de Uso destas Bibliotecas Digitais, disponíveis em https://digitalis.uc.pt/pt-pt/termos.

Conforme exposto nos referidos Termos e Condições de Uso, o descarregamento de títulos de acesso restrito requer uma licença válida de autorização devendo o utilizador aceder ao(s) documento(s) a partir de um endereço de IP da instituição detentora da supramencionada licença.

Ao utilizador é apenas permitido o descarregamento para uso pessoal, pelo que o emprego do(s) título(s) descarregado(s) para outro fim, designadamente comercial, carece de autorização do respetivo autor ou editor da obra.

Na medida em que todas as obras da UC Digitalis se encontram protegidas pelo Código do Direito de Autor e Direitos Conexos e demais legislação aplicável, toda a cópia, parcial ou total, deste documento, nos casos em que é legalmente admitida, deverá conter ou fazer-se acompanhar por este aviso.

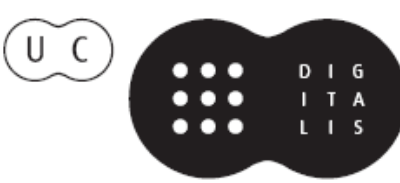



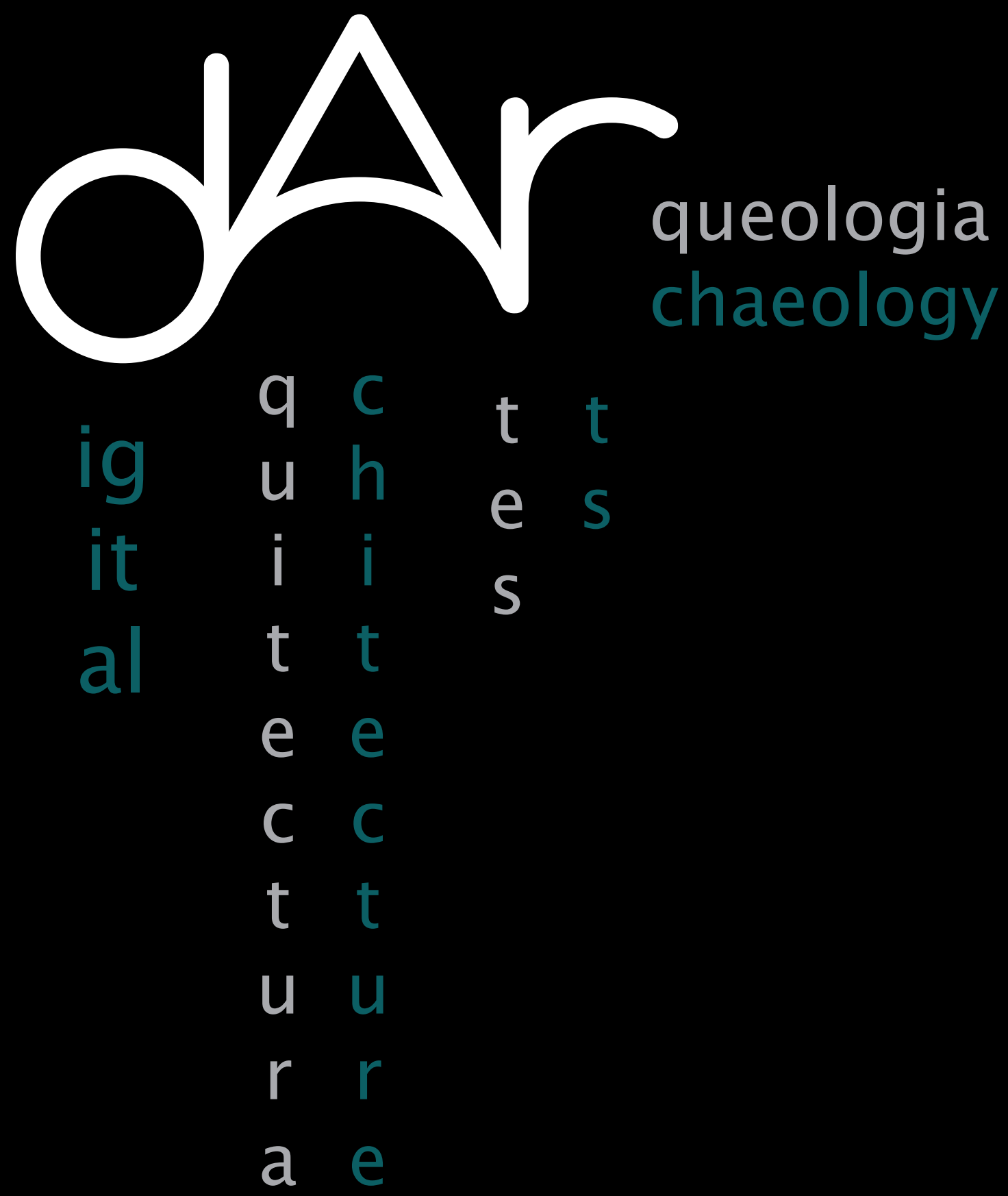

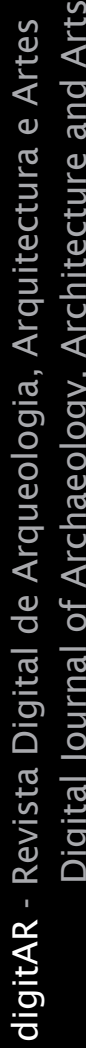




\title{
DÉVELOPPEMENT D'UN ÉCOSYSTÈME TERRITORIAL D'INNOVATION SOCIALE DANS LES ZONES TOURISTIQUES VULNÉRABLES AU MAROC: \\ CAS DES TERRITOIRES OASIENS
}

\author{
Dr. Khadija Askour \\ Institut Supérieur International \\ de Tourisme de Tanger Maroc
}

\section{Résumé}

La vulnérabilité des espaces oasiens au Maroc interpelle les pouvoirs publics sur les alternatives possibles pour un développement territorial durable. Le programme oasis du Tafilalt (POT) initié par le département de l'Aménagement du Territoire en partenariat avec le PNUD s'inscrit dans cette perspective, et propose une démarche de valorisation des filières productives autour de la promotion du produit de terroir. Deux éléments essentiels fondent cette approche à savoir la coopération des acteurs et l'existence de ressources territoriales.

$\mathrm{Au}$ niveau des Oasis, la datte représente la base du développement. Généralement, la valorisation des produits de terroir permet aux territoires « pauvres » ruraux, de sortir de leur marasme économique. L'agriculture de terroir représente un moyen privilégié d'augmentation du revenu chez l'habitant rural. Elle permet, de même, la diminution de l'exode rural en maintenant les populations locales sur place, et la sauvegarde des savoirfaire locaux.

Dans une démarche d'économie solidaire, ce travail de réflexion propose de traiter la problématique du développement des solidarités productives et des solutions innovantes aux problèmes locaux. S'inscrivant dans l'approche marshallienne des districts industriels et des SPL à la française, l'objectif est notamment d'interroger la pertinence de la démarche de coopération proposée par le projet oasis, de conceptualiser l'approche adoptée et d'en définir les modes d'appropriation par les acteurs locaux.

Mots-clés: Système Productif Localisé (SPL), Programme Oasis Tafilalet (POT), Errachidia, Maroc.

https://doi.org/10.14195/2182-844X_5_1 


\section{Introduction}

$\mathrm{Al}^{\prime}$ instar d'un certain nombre de pays, le Maroc s'inscrit clairement dans une démarche publique qui remet en question la pertinence des politiques centralisées. Le défi étant actuellement de proposer aux territoires des possibilités plus larges de développement territorial. Dans ce cadre, la construction d'un écosystème territorial ou SPL représenterait l'outil indispensable pour la résolution des problématiques de développement notamment pour les territoires en marge de développement ou vulnérables.

$\mathrm{Au}$ niveau de notre réflexion, nous allons mettre l'accent sur le développement territorial des Oasis du Tafilalet, représentant l'un des territoires les plus fragiles et en même temps l'un des plus riches en termes de potentialités naturelles.

Nous qualifions notre territoire de recherche à savoir les Oasis comme étant vulnérable. Le sens de la vulnérabilité est définit d'une manière assez générale par la présence d'une faiblesse, d'un manque ou encore d'une déficience. Par rapport au territoire, la vulnérabilité renvoie à l'idée que le lieu fait face à une menace de destruction ou encore d'altération pouvant freiner son fonctionnement et son développement. Dans le contexte des Oasis marqué notamment par une vulnérabilité environnementale et socio-économique, la question du devenir de la filière de la datte reste posée.

\section{De l'espace à la notion de territoire}

Le territoire est aujourd'hui l'une des dimensions les plus mobilisées dans les politiques publiques au Maroc.L'objectif étant d'assurer un développement équilibré entre les territoires marocains économiquement fort et les territoires vulnérables.

La notion de territoire englobe plusieurs dimensions : territoire politique, territoire administratif, territoire de vie, ou territoire de projet. Dans la littérature, le concept de territoire n'est pas l'œuvre d'une seule discipline à savoir la géographie à travers l'espace géographique mais interpelle d'autre horizon disciplinaire comme les sciences économiques à travers l'économie des territoires. Il est considéré comme un espace de structuration de relations de connexions entre agents économiques. Il est également définit comme : " une construction aléatoire d'agents localisés structurés en un réseau de relation » (Rallet, 1993, p.370). Selon Lacour 
(1996), ce concept permet : «d'introduire des dimensions que jusque-là, les économistes avaient grand mal à prendre en compte, notamment les dimensions culturelles, sociales, familiales, religieuses »(Lacour, 1996, p.36).

Cette nouvelle conception de l'espace permet d'approcher le concept de développement territorial.La dialectique territoire/développement fait débat au Maroc depuis plusieurs années auprès de la communauté scientifique (Askour, 2009; Courlet, 2001; Courlet et al., 2006). Les travaux sur la question se sont focalisés essentiellement sur la mise en valeur des ressources territoriales dans une perspective de développement endogène. Un processus de « contagion » semble se dessiner à différentes échelles, passant du scientifique aux actions des politiques publiques. Actuellement, le territoire représentel'échelle d'action la plus prometteuse quant aux retombées souhaitées des actions d'accompagnement de l'Etat marocain. On assiste ainsi à la montée d'une conception nouvelle du développement des territoires qui associe, dans une perspective de croissance économique et de création d'emplois, initiative privée - action publique. Le rôle de l'État s'inscrit notamment dans une logique de « redressement économique et industriel», il intervient face à un contexte marqué par une crise ou le développement et la promotion d'une filière économique particulière.

Toutefois, on retrouve aujourd'hui une panoplie de notions comme : territoire de projet, territoire pertinent ou territoire productif, désignant les mêmes contenues. De même qu'on remarque souvent une confusion des concepts au niveau des outils d'intervention mobilisés et de la démarche qui occultent les composantes du territoire, à savoir : l'activité, la population et l'espace, pour la réduire à une proximité géographique. La reconnaissance du rôle du territoire s'est faite avec l'émergence de formes d'organisations localisées spécifiques dans les pays développés mais également en voie de développement. Le Maroc est passé d'un modèle centré sur une action d'aménagement des sites d'accueil industriel à un système basé sur l'encouragement à des synergies entre acteurs locaux dans la perspective d'impulser un développement territorial.

On rappelle que c'est dans les années 80 , que le territoire apparaît pour la première fois dans la science économique avec l'apport marshallien des districts industriels. Au niveau de ce paradigme, la conception de l'espace limitée à une distance entre les lieux se voit substituer par une conception plus large. 


\section{L'apport marshallien des districts industriels ${ }^{1}$}

Les écrits de l'économiste Alfred Marshall sur les districts industriels représenterait les travaux les plus développés de l'intégration de l'espace en sciences économiques (Duez, 2011).

Alfred Marshall désigna,en 1920, une forme d'organisation industrielle, observable en Angleterre (les petites entreprises du secteur de la coutellerie à Sheffield) comme district industriel. Le district, dans ce cas, tire sa performance des relations non exclusivement marchandes qui existent entre différentes unités de production.

C'est dans l'ouvrage Principes d'économie politique qui date de 1898 au chapitre X du livre IV mais aussi dans Industry and Trade publié en 1919, en particulier au chapitre VI du livre II, qu'A. Marshall traite de la concentration d'industries spécialisées dans certaines localités, qu'il désigne par le terme « d'industrie localisée » (Courlet, 2001). Dans son analyse, il met en avant les économies externes et d'agglomérations (notamment le développement des infrastructures publiques et privées, accès aux inputs et aux marchés, etc.) issues de la localisation d'un certain nombre de firmes en un même endroit. Il nomme, par la suite, ce type de concentration « district industriel ». C'est à dire que l'ensemble de la zone bénéficie d'avantages en termes de coûts de production par le seul fait de la concentration d'activités qui s'y exerce.

Marshall, souligne également, le rôle de la concentration industrielle dans la réalisation de la division du travail, facteur principal de la loi de la productivité croissante. Pour cela, il effectue une lecture croisée de l'organisation économique à travers sa dimension technique et spatiale, et annonce, bien avant que celui-ci apparaisse dans l'analyse économique, la possible existence d'un mode d'organisation efficace qui dévie du clivage marché/hiérarchie (Courlet, 2002, p.42).

Plusieurs éléments ou causes, selon Marshall, ont poussé à la localisation des industries, mais les principales ont été les conditions physiques, telles que : le caractère du climat et du sol, l'existence de mines et de carrières dans le voisinage, l'accès facilité à la terre ou à l'eau. Il donne plusieurs exemples à ce niveau : "Les industries métallurgiques se sont installées généralement près des mines ou dans des lieux où le combustible était bon

\footnotetext{
1 Tirée de l'ouvrage : Askour, K. (2009). Les réseaux de coopération productive au Maroc : le cas de la filière agro-alimentaires, PUM.
} 
marché. Les industries du fer, en Angleterre, cherchèrent d'abord les régions où le charbon de bois était en abondance, et ensuite elles émigrèrent dans le voisinage des houillères. Le Straffordshire fabrique plusieurs espèces de poterie, dont toutes les matières premières sont importées de loin, mais on y a du charbon à bon marché et une excellente terre glaise pour faire les lourds seggars, ou boites dans lesquelles les poteries sont placées pendant qu' on les cuit » (Marshall, 1971, p.462-463).

Contrairement à beaucoup d'autres travaux sur la localisation industrielle, l'analyse de Marshall est originale en la matière, dans le sens où elle accorde peu d'importance aux causes premières exogènes de la localisation. Les éléments énumérés ci-dessus joue un rôle important, tout dépend de l'usage que fait l'homme de ces avantages (Courlet, 2001). Selon Marshall, la diffusion des économies d'organisation renforce la croissance quantitativement et qualitativement (par la diffusion de la nouveauté). Concernant ce deuxième aspect, il écrit en ce sens : « Les secrets de l'industrie cessent d'être des secrets ; ils sont pour ainsi dire dans l'air, et les enfants apprennent inconsciemment beaucoup d'entre eux [...]. Si quelqu'un trouve une idée nouvelle, elle est aussitôt reprise par d'autres, et combinée avec des idées de leur crû ; elle devient ainsi la source d'autres idées nouvelles. Bientôt des industries subsidiaires naissent dans le voisinage, fournissant à l'industrie principale les instruments et les matières premières, organisant son trafic, et lui permettant de faire bien des économies diverses. » (Marshall, 1971, p.465).

Deux grandes catégories d'avantages retiennent l'attention de Marshall. Il s'agit d'une part des avantages liés à l'existence et la constitution d'un marché local pour un même type de qualification des ressources humaines. Ces avantages renvoient, à la coopération des forces sociales avec les forces économiques, ce que Marshall appelle une « atmosphère industrielle »: «Une industrie localisée tire un grand avantage du fait qu'elle est constamment un marché pour un genre particulier de travail. Les patrons sont disposés à s'adresser à un endroit où ils ont des chances de trouver un bon choix d'ouvriers possédant les aptitudes spéciales qu'il leur faut ; de leur côté les ouvriers cherchant du travail vont naturellement dans ces endroits où se trouvent beaucoup de patrons ayant besoin d'ouvriers de leur spécialité et où ils ont, par suite, des chances de trouver un marché avantageux. " (ibid. p.466). Cette atmosphère, qui englobe une proximité géographique et une spécialisation dans une même branche, permet l'innovation et le développement de nouvelles entreprises et favorise l'échange d'idées et d'expériences concernant les entreprises et la branche (Mathot, 2001) 
Le deuxième type d'avantage tire ses fruits de l'interdépendance technique des activités créées par la naissance, "dans le voisinage » d'une industrie principale, d'industries auxiliaires situées techniquement en amont et en aval de celle-ci, au sein d'une même " filière productive » (Courlet, 2001). D'après Marshall, une région qui vit d'un seul type d'activité, pourrait être exposée à une crise très grave. Dans ce sens, il note : " Une industrie localisée offre quelques inconvénients, en tant que marché de travail, si, dans le travail qui s'y fait, une seule espèce prédomine, par exemple, un travail ne pouvant être exécuté que par des hommes très forts. Dans les régions métallurgiques où il n'y a ni industrie textile, ni aucune autre industrie pour donner du travail aux femmes et aux enfants, les salaires sont élevés, et le travail est coûteux pour le patron, bien que les revenus moyens en monnaie de chaque famille soient bas. Mais le remède à ce mal est évident, et il est fourni par le développement dans la même région d'industries d'un caractère supplémentaire. » (Marshall, 1971, p.467). Ces activités permettent d'obtenir des avantages qui se combinent avec ceux de la localisation de l'industrie dans certaines localités des grandes villes manufacturières, et c'est selon Marshall, l'une des principales causes de leurs progrès continus.

Les économies externes sont marquées par un ancrage territorial chez Marshall. Elles ont une forte irréversibilité reposant sur les structures historiques et sociales d'un district. Le district est une construction à partir d'avantages créés et non innés (Gaffard et Romani, 1990)². Dans cette perspective, l'efficacité d'un système localisé d'entreprises, serait, dans une large mesure, le résultat de son inscription socio- territoriale.

\section{Les SPL au Maroc, un concept à interroger}

Les SPL, les districts industriels ou encore les clusters sont devenus la pierre de lance des politiques de développement des territoires notamment dans les pays en voie de développement comme le Maroc. Les enjeux auxquels les territoires sont confrontés aujourd'hui, appellent à une reconfiguration des relations entre entreprises sous la forme de réseaux. Les synergies constituent ainsi, comme le montrent bon nombre d'exemples étrangers (districts industriels italien), une issue vers la réussite économique. Benko et lipietz (2000) parlent, dans ce cas, de territoires gagnants.

Un débordement existerait néanmoins sur le plan des concepts «approuvés »

2 Cité par Courlet C. (2002, p.29) 
par la littérature scientifique ! Le SPL, l'écosystème territorial, le district industriel, le district technologique, les milieux innovateurs, les aires de spécialisation, sont autant de notionsqui rendent difficile la détermination des contours réels des phénomènes de coopération localisée au Maroc et ailleurs.

Tous ces réseaux de collaboration sont des variantes du système productif localisé(SPL) mais ont toutefois des caractéristiques similaires dansl'approche retenue pour les catégoriser à savoir une proximité organisationnelle et une proximité institutionnelle.

Courlet définit le SPL comme: « une configuration d'entreprises regroupées dans un espace de proximité autour d'un métier, voire de plusieurs métiers industriels ou tertiaires. Les entreprises entretiennent des relations entre elles et avec le milieu socio-culturel d'insertion. Ces relations ne sont pas seulement marchandes, elles sont aussi informelles et produisent des externalités positives pour l'ensemble des entreprises. Le métier industriel dominant n'exclut pas la possibilité de l'existence (au sein d'un SPL) de plusieurs branches industrielles. Souvent, on fait référence à des systèmes de PME ; cependant, il existe aussi des relations très territorialisées entre grandes entreprises et PME (dans un rapport autre que celui de la soustraitance traditionnelle)» (Courlet, 2001).

LeSPL permet, un ancrage territorial, de même qu'il représente généralement un catalyseur du développement et de la performance économique des entreprises qui le forment. L'exemple italien montre que l'appartenance à un district accroit la rentabilité des entreprises de 2 à $4 \%$, en moyenne, du fait des économies d'agglomération créées par les SPL (OCDE., DATAR, 2001, p.8). Les entreprises d'un SPL profitent de bassins de main d'œuvre spécialisée, de services aux entreprises et d'idées, etc. Ce qui n'est pas le cas d'entreprises isolées les unes des autres.

$\mathrm{Au}$ Maroc, le système productif local représente le concept le plus sollicité par l'intervention des pouvoirs publics dans le cadre des politiques de développement territorial. Depuis les années 90, l'Etat marocain donne une importance à la promotion des PME, qui représente plus de $90 \%$ du tissu productif, notamment avec des mesures et actions d'appui et d'assistance à la « construction » des réseaux de coopération. On constate ainsi la volonté $\mathrm{d}$ 'impulser une dynamique territoriale et endogène. A titre d'exemple, $l^{\prime}$ action conjointe du ministère du commerce et de l'industrie en collaboration avec l'ONUDI proposa aux acteurs locaux une meilleure organisation de la filière de production ainsi que de la chaîne de valeur. C'est le cas des filières à faible rentabilité comme le thuya à Essaouira (Askour, 2009). 
Les SPL donnent ainsi au territoire un nouveau rôle. Dans une économie de plus en plus globalisée, marquée par la concurrence internationale, la proximité des individus et/ou des firmes dans un espace déterminé, représente la composante essentielle du processus de développement local. Toutefois, si l'on considère le caractère socio-économique du SPL, on constate clairement qu'au Maroc, l'identité culturelle ou encore les valeurs locales, sont évincées dans les critères d'identification et de construction de SPL. En effet, ce concept projette le développement d'espaces identifiés selon des indicateurs qui ne répondent pas toujours aux prérequis de la notion avancée.L'identification des agglomérations porte sur des lieux où une activité est exercée dans 5 établissements productifs au moins; représente au niveau local 100 emplois au moins; représente $5 \%$ au moins de l'emploi de l'activité au niveau du Maroc; et enfin représente 5\% au moins de l'emploi industriel local. Sur cette base, il est identifié un modèle chiffré d'organisation industrielle localisée, éventuel outil des politiques publiques mais ne remplissant que partiellement les caractéristiques propres à un SPL. Ainsi, on peut dire que les configurations productives territoriales qui émergent au Maroc, sont « issues », d'une politique volontariste étatique d'agglomération et de polarisation des activités économiques qui adopte le concept SPL sans pour autant l'adapter. Ce qui aboutit de loin aux résultats escomptés.

En parallèle à ce mouvement, des initiatives locales issues de la volonté propre des populations autochtones apparaissent. Ces comportements relationnels de coopération locale dits " réussis » sont issus des volontés de solidarité et d'entraide entre les populations d'une même localité. On les identifie au niveau de certaines régions du Maroc comme le Souss Massa, parmi les arrangements sociaux des sociétés tribales. Les institutions traditionnelles ont été reprises dans les nouvelles configurations productives, avec l'émergence des coopératives productives (Askour, 2009).Au niveau des Oasis de Tafilalet, la présence de l'héritage solidaire ancestral et des liens forts au territoire représente le gage de réussite de la mise en place d'un SPL.

\section{L'écosystème territorial oasien}

En partant des réalités économiques, on remarque que souvent les entreprises localisées dans des milieux fragiles au Maroc aussi diverses soient-elles interagissent avec leur territoire d'appartenance. Le développement des territoires est réalisé ainsi à travers un choix voulu et non imposé des entreprises.

La collaboration inter-entreprises offre aux entreprises des opportunités 
d'évolution, ce qui engendre une dynamique territoriale, permettant de dépasser des situations de crise ou de menace notamment pour les territoires fragiles. Cette situation représente, comme l'indique Bernard Pecqueur ${ }^{3}$, une alternative au développement des territoires. C'est le cas des entreprises localisées dans des territoires riches en ressources locales mais pauvres en activité économique.

L'appartenance à des réseaux augmenterait même de manière significative la capacité à innover, et ce en donnant la possibilité d'accès à de nouvelles informations et ressources (Guihur, Julien et Trépanier, 2009).

La formule la plus sollicitée par les pouvoirs publics au Maroc afin de redynamiser ces territoires en "manque de développement " se fonde sur l'organisation et la valorisation des filières sous la forme d'agglomération et de mise en réseau. La production et la commercialisation des produits de terroir sont pour ces territoires, une aubaine de développement. L'encouragement aux agglomérations proposé, dans ce cas, adhère au modèle du SPL qui marque la banalisation de l'entreprise individuelle. Dans ce cas, l'État n'intervient que comme le catalyseur de la dynamique, on est en face d'un nouveau modèle de développement, l'État accompagnateur ! Le Maroc est composé de plusieurs régions dont les spécificités socioéconomiques et géographiques se diffèrent d'une manière importante. Les territoires du Sud et de l'arrière-pays du Maroc sont loin d'être des territoires compétitifs. C'est notamment leurs ressources naturelles qui leur permettent de surpasser les phénomènes d'enclavement économique. C'est le cas des Oasis de Tafilalet au niveau d'Errachidia. Cette province est connue par ses oasis et ses dunes de sable, et s'étale sur une superficie de $60000 \mathrm{~km} 2$ au Sud est marocain, avec une population de 556612 habitants dont $65 \%$ résident en milieu rural et $35 \%$ en milieu urbain. Sur le plan économique, le Tafilalet reste une région principalement agricole notamment avec la production de l'huile d'olive et des dattes.

Depuis plusieurs siècles, l'économie des régions sub-sahariennes du Sud du Maroc repose sur l'exploitation agricole des palmiers dattiers. La production des dattes dans la province représente environ $27 \%$ de la production nationale. Cette production reste toutefois menacée par le fléau du Bayoud et par les longues saisons de sécheresse.

L'évolution de la filière de la datte s'est faite par une modernisation

\footnotetext{
3 Pecqeur, B. et al., (1996). Dynamique territoriale et mutations économiques, Paris, L’Harmattan.
} 
des pratiques agricoles (irrigation à l'aide de motopompes, etc.) et par le développement d'une activité connexe à savoir l'activité touristique. Le tourisme à Errachidia se base sur des potentialités naturelles, géographiques et socio-historiques importantes, on cite notamment les ksour et Casbahs, les oasis, le désert, le patrimoine culturel, les paysages de montagne.

Toutefois, de par les phénomènes de désertification et de dégradation, la situation des oasis est aujourd'hui plus que préoccupante. Face à cette urgence, les pouvoirs publics marocains ont mis en place un programme de développement territorial durable des oasis du Tafilalet. Ce programme propose la construction et la mise en place d'un écosystème désertique dunaire sous la forme d'un SPL (la route de Majhoul en représente l'une des composantes essentielles,) et d'un SPL touristique à Merzouga.

La route du Majhoul (RM) représente une alternative pour le développement de l'écotourisme oasien. Il s'agit d'un circuit touristique thématique à l'origine qui a évolué vers une marque touristique territoriale à partir duquela vu le jour un réseau d'acteurs locaux qui se sont constitués en association de promoteurs de l'écotourisme puis en Groupement d'Intérêt Economique pour exercer une activité touristique.

LeSPL de Merzouga, quantà lui s'articule autour de deux axes fondamentaux: le zoning touristique et la signalétique touristique. Le premier axe consiste a divisé le territoire touristique de Merzouga en des zones, chacune étant consacré par une activité. Le second axe porte sur l'unification des panneaux de signalisation afin de lutter contre la pollution visuelle.

\section{Le SPL de la Route du Majhoul}

L'analyse d'un SPL se fait par l'intersection entre deux types de proximité : la proximité géographique et la proximité organisationnelle, mais elle peut également impliquer un troisième type de proximité à savoir institutionnelle (Bellet, Colletis et Lung, 1993, p.357).

La première proximité dite aussi fonctionnelle renvoie à un espace géonomique. Toutefois, elle ne se limite pas à une proximité physique dans la mesure où elle n'est pas donnée par les contraintes naturelles mais elle est construite socialement. Sur la route du Majhoul, s'étalant sur la vallée de Ziz et Ghris passant par la commune d'Errachidia, Frekla l'oulia et Taouz, on constate une proximité géographique de l'ensemble des acteurs du SPL

Le deuxième type de proximité traduit la séparation économique entre 
les agents, les individus, les différentes organisations et/ou institutions. Elle dépend essentiellement des représentations en fonction desquelles les agents inscrivent leurs pratiques (stratégies, décisions, choix, etc.), et concerne les relations interindividuelles notamment la dimension collective, à l'intérieur des organisations ou entre les organisations. La proximité organisationnelle a plusieurs dimensions. Elle peut être appréhendée sur le plan technologique, industriel ou financier. Sur la route du Majhoul, les coopérations des acteurs se caractérisent par un mode de coopération formel et permanent dans la majorité des cas. Par contre les relations se limitent à des relations privé-privé. Les collectivités locales plus particulièrement les communes d'Errachidia et Frekla Oulia, $\mathrm{n}^{\prime}$ interviennent en aucun cas dans le développement du SPL de la route du Majhoul.

Le troisième type de proximité à savoir institutionnelle, peut être défini comme un facteur explicatif des performances de croissance des systèmes économiques. La notion d'écosystème désertique dunaire, à titre d'exemple, traduit bien l'idée selon laquelle l'appartenance ou le recours à un même ensemble institutionnel peut favoriser le développement économique. Cet ensembleinstitutionnelenglobe une multitude de dimensions: infrastructures éducatives, infrastructures de recherche publique, établissements financiers, et d'une manière plus élargie les politiques publiques intervenant dans le domaine. Dans le cas des SPL, cette proximité est étroitement liée avec la proximité géographique, lorsque des pratiques locales permettent la mise en commun de ressources pour créer un bien public spécifique, une atmosphère industrielle (Marshall, 1919).

La délégation du tourisme, l'Inspection de l'aménagement du Territoire ou encore le POT (Programme Oasis Tafilalet), ont un rôle certes très présent et important dans l'élaboration de la stratégie de l'écotourisme au niveau du Tafilalet mais ne garantit en rien la pérennité du SPL.

La réussite du SPL de la route du Majhoul revient largement au fait que l'ensemble de la population locale a adhéré au projet. Les acteurs locaux entretenaient néanmoins déjà des relations assez fortes avec leur territoire d'appartenance avant l'application de la stratégie des pouvoirs publics.

\section{Conclusion}

${ }^{4}$ Cité par Colletis G. et Rychen F. (2004, p.221). 
L'analyse de la littérature d'un ensemble de théoriciens de disciplines différentes comme les économistes, les géographes, les sociologues ou les historiens, traitant des formes de réseautage d'entreprises, permet deconstater une utilisation massive de la notion de SPL. LeSPL se distingue ainsi par deux caractéristiques fondamentales à savoir une proximité organisationnelle et une proximité géographique et dans certains cas, institutionnelle. Au niveau du territoire oasien, les dynamiques locales s'apparentent parfaitement à ce que l'on pourrait nommer un «SPL à la marocaine », de par la présence de coopérations formelles et informelles ainsi que de la proximité géographique et institutionnelle existante entre les acteurs locaux.

D'une manière générale, le SPL a comme qualité d'être un moyen efficace pour améliorer la compétitivité des entreprises qui s'associent pour atteindre des économies d'échelle. Toutefois, au niveau des Oasis de Tafilalet, il est davantage une nécessité plus qu'un choix. Il est le résultat d'une crise du fordisme et de la présence de situation de pauvreté de la population locale. Le SPL oasien représente ainsi une alternative pertinente pour ce type de territoires fragiles et vulnérables en permettant de renforcer les liens de solidarité déjà existants, et d'inscrire le territoire dans une logique de développement territorial.

\section{Bibliographie}

Askour, K. (2009). Les réseaux de coopération productive au Maroc : le cas de la filière agro-alimentaire, PUM.

Bellet M., Colletis G., Lung Y. (1993), "Economie de proximité »", in Revue $\mathrm{d}^{\prime}$ Economie Régionale et Urbaine, $\mathrm{n}^{\circ} 3$.

Benko G., Lipietz A. (2000), "Géographie socio-économique ou économie géographique ? », in Benko G., Lipietz A. (dir), La richesse des régions- la nouvelle géographie socioéconomique, coll. « Economie en liberté », PUF, Paris.

Courlet, C. (2001). "Les systèmes productifs localisés : un bilan de littérature ", inles Cahiers d'Economie et Sociologie Rurales, Paris, Institut de la Recherche Agronomique, $n^{\circ} 58-59$.

Courlet C. (2002), "Les Systèmes productifs Localisée : un bilan de littérature ", in Etudes et Recherches sur les Systèmes Agraires et le Développement, Le local à l'épreuve de l'économie spatiale: Agriculture, environnement, espaces ruraux, n³3, INRA.

Courlet, C. et al. (2006). Territoire et développement économique au Maroc : Le cas 
des systèmes productifs localisés, in Courlet C. (dir), coll. " Economie critique », l'Harmattan.

DATAR., OCDE. (2001), Actes du congrès mondial des systèmes productifs locaux, Paris, les 23-24 janvier.

Guihur, I., P.-A. Julien et M. Trépanier (2009). Le processus d'innovation en réseau dense : autopsie d'un échec en co-développement. Communication présentée à la 11 eme journée scientifique du réseau entrepreneuriat, Canada.

Lacour C., (1996), « La tectonique des territoires : d'une métaphore à une théorisation », in Pecqueur B., Dynamiques territoriales et mutations économiques, L'Harmattan, Paris, pp.25-48.

Marshall A. (1971), Principe d'économie politique, Traduction en langue française, Tome 1, Livre IV, chp.10.

Mathot P. (2001), Districts industriels et systèmes locaux de production, note de synthèse.

Philippe Duez, «La place de l'économie des territoires dans la construction d'une théorie générale intégrant l'espace », Revue d'Économie Régionale \& Urbaine 2011/ 4 (octobre), p. 735-764. 